\title{
Modulation of mu attenuation to social stimuli in children and adults with $16 p 11.2$ deletions and duplications
}

Caitlin M. Hudac ${ }^{1 \dagger}$, Anna Kresse ${ }^{2 \dagger}$, Benjamin Aaronson ${ }^{1}$, Trent D. DesChamps ${ }^{1}$, Sara Jane Webb ${ }^{1,2}$ and Raphael A. Bernier ${ }^{1 *}$

\begin{abstract}
Background: Copy number variations (CNV) within the recurrent $~ 600 \mathrm{~kb}$ chromosomal locus of $16 p 11.2$ are associated with a wide range of neurodevelopmental disorders, including autism spectrum disorder (ASD). However, little is known about the social brain phenotype of 16p11.2 CNV and how this phenotype is related to the social impairments associated with CNVs at this locus. The aim of this preliminary study was to use molecular subtyping to establish the social brain phenotype of individuals with 16p11.2 CNV and how these patterns relate to typical development and ASD.

Methods: We evaluated the social brain phenotype as expressed by mu attenuation in 48 children and adults characterized as duplication carriers $(n=12)$, deletion carriers $(n=12)$, individuals with idiopathic ASD $(n=8)$, and neurotypical controls $(n=16)$. Participants watched videos containing social and nonsocial motion during electroencephalogram (EEG) acquisition.

Results: Overall, only the typical group exhibited predicted patterns of mu modulation to social information (e.g., greater mu attenuation for social than nonsocial motion). Both 16p11.2 CNV groups exhibited more mu attenuation for nonsocial than social motion. The ASD group did not discriminate between conditions and demonstrated less mu attenuation compared to the typical and duplication carriers. Single-trial analysis indicated that mu attenuation decreased over time more rapidly for 16p11.2 CNV groups than the typical group. The duplication group did not diverge from typical patterns of mu attenuation until after initial exposure.
\end{abstract}

Conclusions: These results indicate atypical but unique patterns of mu attenuation for deletion and duplication carriers, highlighting the need to continue characterizing the social brain phenotype associated with 16p11.2 CNVs.

Keywords: 16p11.2, Copy number variation (CNV), Autism spectrum disorder (ASD), Mu attenuation, Electroencephalogram (EEG), Social perception, Molecular subtyping

\section{Background}

Autism spectrum disorders (ASD) are associated with a recurrent $\sim 600 \mathrm{~kb}$ BP4-BP5 16p11.2 copy number variation $(\mathrm{CNV})[1-4]$. The overall population prevalence of 16p11.2 deletions and duplications is estimated at $1 / 1000$ [5], but their prevalence is much higher in clinical populations, with both types of CNVs accounting for approximately $1 \%$ of ASD cases [1,2]. Additional clinical features of 16p11.2 CNV include congenital anomalies,

\footnotetext{
* Correspondence: rab2@u.washington.edu.edu

${ }^{\dagger}$ Equal contributors

'Department of Psychiatry and Behavioral Sciences, University of Washington, 1959 Northeast Pacific Street \#115, Seattle, WA 98195, USA Full list of author information is available at the end of the article
}

language delay, epilepsy/seizures, and behavioral problems such as attention deficit hyperactivity disorder [3, 5-14]. Notably, $15 \%$ of deletion carriers meet strict diagnostic criteria for ASD [15]. Even in individuals not meeting criteria for behaviorally defined ASD diagnoses, 16p11.2 $\mathrm{CNV}$ confers a quantitative risk to a variety of phenotypic domains [16] and specifically to social ability [15, 17], with an observed $1.7 \mathrm{SD}$ decrement in social ability relative to unaffected family members.

Given this evidence of social impairments associated with 16p11.2 CNV and the hallmark social impairments in ASD $[3,5-14,18]$, the social brain is an appropriate target to elucidate the relationship between genetics and 
social functioning. Perception of social motion (e.g., biological motion) has been suggested as an ideal candidate neuroendophenotype $[16,19]$, and social motion perception may be a critical process that underlies imitation, the interpretation of goals, and social-action understanding $[20,21]$. Evidence using neuroimaging suggests that individuals with ASD exhibit atypical social motion perception, including reduced activation compared to controls within the superior temporal sulcus, fusiform gyrus, and prefrontal cortex [1-4, 19, 22, 23].

Many children with neurodevelopmental disorders, including children with 16p11.2 may struggle in the fMRI scanning environment, and as such, electroencephalogram (EEG) provides a more behaviorally flexible means of assessing social neural systems. EEG mu attenuation is one such measurement of social brain function that is helpful in understanding how social difficulties relate to underlying brain function. $\mathrm{Mu}$ attenuation is computed as power changes in the electrophysiological mu rhythm band $(8-13 \mathrm{~Hz})$ in response to observed motion. Greater $\mathrm{mu}$ attenuation is thought to reflect desynchronization of cell assemblies in sensorimotor cortex [5, 24]. Typically developing individuals elicit mu attenuation by the execution and observation of motoric action [1, 2, 24-30]. Such findings are thought to be evidence of an execution/observation matching system $[3,5-14,31]$ that contributes to many facets of social cognition including imitation, empathy, and perception of goal-directed actions [15, 32-34]. Impairments of this system have been associated with ASD and heterogeneity within the ASD diagnosis [5, 16, 35-38], suggesting that it may be sensitive to the deficits in social ability in 16p11.2 CNVs.

The aim of this study was to better characterize the functional brain phenotype of a preliminary sample of 16p11.2 CNV carriers using mu attenuation elicited by videos of social and nonsocial motion. First, group differences were evaluated between typical individuals, $16 p 11.2$ deletion carriers, 16p11.2 duplication carriers, and individuals identified with idiopathic autism. Overall, we predicted that the 16p11.2 CNV groups would exhibit atypical mu attenuation similar to the ASD group. Second, in addition to overall group patterns, we aimed to determine how patterns of mu attenuation changed over the course of stimulus exposure for each group. Recent evidence suggests that mu attenuation may progressively change over the course of an experiment $[1,2,15,17,39]$. Despite known differences in information processing $[3,5-14,18,40-42]$ and neural evidence of reduced habituation in ASD to social stimuli $[15,16,19,43,44]$, no studies have explored how mu attenuation is modulated over time in ASD. Single-trial analyses were implemented with the expectation that habituation patterns would differ between the 16p11.2 CNV groups, the ASD group, and the typical individuals. Lastly, post hoc comparisons were included to better characterize patterns of mu attenuation in the 16p11.2 CNV groups, considering the heterogeneity related to age and ASD diagnosis.

\section{Methods \\ Participants}

This study reports findings from 48 child and adult participants who were enrolled in and characterized as belonging to one of four groups: (1) deletion carrier, (2) duplication carrier, (3) idiopathic ASD, and (4) typical development. All participants spoke fluent English and had normal or corrected-to-normal vision. All research procedures conformed to regulations in accordance with the local ethical review board at the University of Washington, which approved this project. Written informed consent was obtained from each adult participant or parental representative(s). All children verbally assented to participate in the procedures, and written assent was obtained from children with a mental age of 7 or greater.

Full participant characterization is reported in Table 1. Deletion $(n=12)$ and duplication $(n=12)$ carriers were recruited following enrollment and participation in the Simons VIP Connect project [16, 20, 21, 45]. Recruitment methods included self-referral and web-based networks that directed individuals to the Simons VIP website (http://SimonsVIPconnect.org), as well as referral by clinical genetic testing centers. As part of the Simons VIP Connect, individuals were identified with the same recurrent $\sim 600 \mathrm{~kb} 16 \mathrm{p} 11.12 \mathrm{BP} 4-\mathrm{BP} 5$ deletion or duplication. Within these two 16p11.2 CNV groups, no additional pathogenetic $\mathrm{CNVs}$ or monogenic disorders were known (see Simons VIP Consortium [45] for complete recruitment and inclusion/exclusion criteria). Individuals within the ASD and typical groups were recruited from previous projects, matched on age (ASD/typical) and verbal IQ (ASD) to the CNV groups. None of the children within the idiopathic ASD group had any likely causal CNVs, verified through array CGH sequencing $[15,17,46]$.

For ASD and 16p11.2 CNV groups, diagnosis of ASD was confirmed using the Autism Diagnostic InterviewRevised [3, 5-14, 18, 47] and the Autism Diagnostic Observation Schedule [16, 19, 48]. IQ was assessed using the Wechsler Abbreviated Scale of Intelligence $[20,21,49]$ or the Differential Ability Scales-Second edition $[1-4,19,22$, $23,50]$, depending on age. One-way analysis of variance (ANOVA) indicated that there were no differences between groups in age, $F(3,44)=1.59, p=0.21$. The duplication, deletion, and the ASD groups did not differ in verbal IQ or SRS total score, $F_{\mathrm{s}}(2,29)<2.96, p \mathrm{~s}>0.068$, but did differ in full-scale and nonverbal IQ, $F_{\mathrm{S}}(2,29)>3.68, p \mathrm{~s}<0.038$. Least squares post hoc comparisons indicated that the ASD group had higher full-scale IQ and nonverbal IQ than both 16p11.2 deletion (FSIQ, $\mathrm{SE}=22.14, p=0.027$; NVIQ, 
Table 1 Participant characterization

\begin{tabular}{|c|c|c|c|c|c|c|c|c|c|c|c|}
\hline Group & $N$ & $\begin{array}{l}\text { Age mean } \\
\text { in years (SD) }\end{array}$ & $\begin{array}{l}\text { Age range } \\
\text { (years) }\end{array}$ & $\begin{array}{l}\text { Adult: } \\
\text { children }\end{array}$ & Gender & $\begin{array}{l}\text { ASD } \\
\text { diagnosis }\end{array}$ & $\begin{array}{l}\text { FSIQ mean } \\
\text { (SD) }\end{array}$ & $\begin{array}{l}\text { VIQ mean } \\
\text { (SD) }\end{array}$ & $\begin{array}{l}\text { NVIQ mean } \\
\text { (SD) }\end{array}$ & $\begin{array}{l}\text { SRS total } \\
(\mathrm{SD})^{\mathrm{a}}\end{array}$ & $\begin{array}{l}\text { CNV } \\
\text { inheritance }\end{array}$ \\
\hline \multirow{4}{*}{$\begin{array}{l}\text { Deletion } \\
\text { carriers }\end{array}$} & 12 & $18.1(14.0)$ & $8-45$ & $3: 9$ & 4 males & 3 yes & $88.36(17.73)$ & 86.00 (18.66) & 91.75 (17.43) & $70.36(17.10)$ & 4 de novo \\
\hline & & & & & 7 females & 9 no & & & & & 4 inherited \\
\hline & & & & & & & & & & & $\begin{array}{l}1 \text { germline } \\
\text { mosaicism }\end{array}$ \\
\hline & & & & & & & & & & & 3 unknown \\
\hline \multirow{3}{*}{$\begin{array}{l}\text { Duplication } \\
\text { carriers }\end{array}$} & 12 & 22.7 (13.8) & $6-50$ & $5: 7$ & 6 males & 3 yes & $87.00(23.31)$ & $87.92(24.30)$ & $87.42(21.36)$ & $67.17(17.7)$ & 1 de novo \\
\hline & & & & & 6 females & 9 no & & & & & 7 inherited \\
\hline & & & & & & & & & & & 4 unknown \\
\hline \multirow[t]{2}{*}{ ASD } & 8 & $11.5(3.3)$ & $7-15$ & $0: 8$ & 4 males & 11 yes & $110.5(18.97)$ & $107.25(15.17)$ & 110.88 (20.06) & 77.50 (12.84) & - \\
\hline & & & & & 4 females & & & & & & \\
\hline \multirow[t]{2}{*}{ Typical } & 16 & $16.2(10.5)$ & $8-43$ & $4: 12$ & 9 males & 16 no & $119.56(7.11)$ & $121.63(10.15)$ & $113.25(8.58)$ & $44.00(3.79)$ & - \\
\hline & & & & & 7 females & & & & & & \\
\hline
\end{tabular}

One deletion carrier excluded from FSIQ and VIQ characterization due to English as second language. One duplication carrier IQ scores reported as a ratio between mental and chronological age because standard scores were unavailable

ASD autism spectrum disorders, SD standard deviation, FSIQ full-scale IQ, VIQ verbal IQ, NVIQ nonverbal IQ, CNV copy number variation, SRS social responsiveness scale

${ }^{\mathrm{a}}$ Missing data from deletion carrier $(n=1)$, typical group $(n=4)$

$\mathrm{SE}=19.13, p=0.041)$ and $16 p 11.2$ duplication (FSIQ, $\mathrm{SE}=23.50, \quad p=0.018 ; \quad \mathrm{NVIQ}, \quad \mathrm{SE}=23.46, \quad p=0.014)$ groups. None of the typical participants had elevated scores on the SRS nor met diagnostic criteria for any psychiatric disorders.

\section{Social motion task}

Participants observed a series of silent 1-min-long videos. Stimuli consisted of three conditions: (1) the social motion condition included a video of a faceless computergenerated (CG) avatar dancing and a live action (LA) video of two pairs of hands engaged in a clapping game. The computerized dancer stimuli were provided courtesy of Nick Neave and Kristofor McCarty at Northumbria University. (2) The nonsocial motion condition included a video of a CG bouncing ball and a LA video of cardboard tubes swinging. (3) The rest condition consisted of static image of the same background as the videos (Fig. 1). The quantity of visual movement was controlled across social and nonsocial motion conditions. Background complexity was controlled across all conditions. All videos were presented twice, so that each participant observed eight 1min movement videos (four social, four nonsocial) and four 1-min rest videos. All participants saw the LA videos first followed by the CG videos, and the order of video context (i.e., social or nonsocial) was counterbalanced across participants (see Fig. 1 for possible presentation orders). Between videos, participants were directed to take a break and the experimenter initiated the next stimulus after confirmation that the participant was ready. Participants were seated approximately $75 \mathrm{~cm}$ from a video monitor and were instructed to sit still and attend to the videos. Video stimuli were displayed using E-Prime 2.0 software (Psychology Software Tools, Inc. Pittsburgh, PA) at a size of $27 \mathrm{~cm}$ by $36.8 \mathrm{~cm}$ and subtended a visual angle of $20.4^{\circ}$ by $27.6^{\circ}$.

\section{Electrophysiological recording and preprocessing}

Continuous EEG was recorded from a high-density 128-channel geodesic net using Net Station 4.3.1 software integrated with a 200-series high-impedance amplifier (Electric Geodesics Inc, Eugene, OR). Electrode impedances were below $50 \mathrm{k} \Omega$ to maximize signal-to-noise ratio, within the standard range for high-impedance amplifiers. During collection, EEG signals were referenced to the vertex electrode, analog filtered $(0.1 \mathrm{~Hz}$ high-pass, $100 \mathrm{~Hz}$ elliptical low-pass), amplified, and digitized with a sampling rate of $500 \mathrm{~Hz}$. A photocell recorded and marked the precise onset time of each video. During acquisition, researchers observed and marked periods containing movement and/or improper attention (e.g., participant looking away). As electrodes surrounding the face, eyes, and rim of the net are prone to artifacts, we excluded these electrodes in all participants from all subsequent post-processing or analyses (see Fig. 2 for locations).

Following data collection, data was filtered using a high-pass filter of $1 \mathrm{~Hz}$. Continuous EEG was segmented into 2-s epochs starting with the onset of each 1-min video (as marked by the photocell). Epochs marked during acquisition as contaminated by movement or improper attention were removed. Automatic 


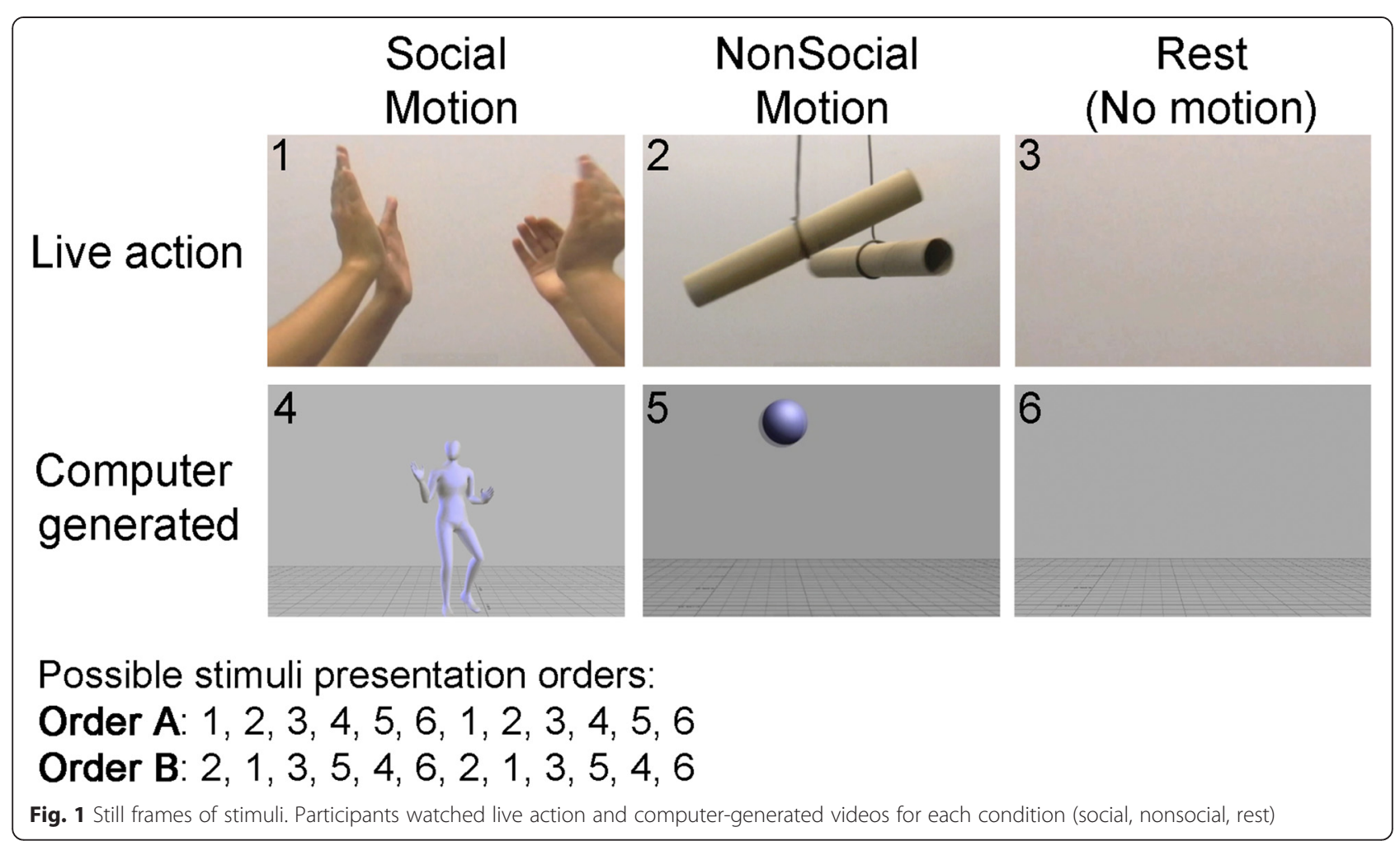

artifact detection rejected channels containing voltage shifts greater than $100 \mu \mathrm{V}$ for each epoch. If the channel was rejected for more than $50 \%$ of epochs, the channel was excluded from analysis. Epochs were rereferenced to the average reference and exported as a

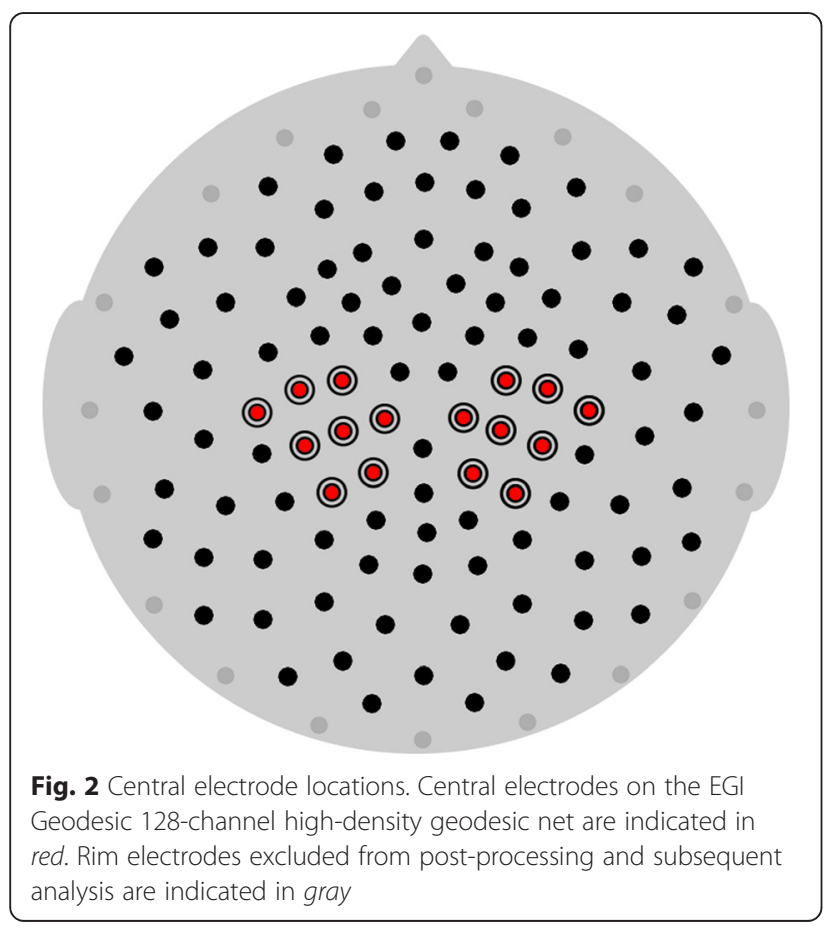

concatenated file per participant for additional artifact rejection using independent components analysis (ICA) via EEGLAB 13.2.1 [5, 24, 51]. First, the ICA runica algorithm was implemented across the entire concatenated dataset for each participant. Epochs related to artifacts were rejected manually. A second ICA with the runica algorithm was implemented to reject components related to artifacts. The resultant primary independent components (i.e., first 35) containing horizontal and vertical eye and/or body movements were manually inspected. Independent components containing artifacts were rejected and removed from the data. The remaining data for each condition was segmented into 2-s epochs for analysis.

To calculate mu power, fast Fourier transforms (FFTs) were conducted in Matlab (version 7.12.0, R2011a; Natick, MA) on each 2-s epoch. The power spectra occurring between 8 and $13 \mathrm{~Hz}$ was averaged across central electrodes clustered around the standard C3 (electrode 37) and C4 (electrode 104) positions (see Fig. 2 for locations). Power spectra for each epoch per condition were considered relative to the average rest condition power. $\mathrm{Mu}$ attenuation was computed as the natural log of the ratio between power for each epoch of either social or nonsocial motion minus the average power of the rest condition for that individual. Subsequently, zero represents no mu attenuation (social motion - rest $=0$ ) and larger negative values represent more mu attenuation (i.e., social motion $<$ rest). 


\section{Data analysis strategies}

All analyses were conducted via SAS 9.3 (SAS Institute) using restricted maximum likelihood (REML) and Satterthwaite denominator degrees of freedom. To fully assess group condition differences, multilevel models were generated using PROC MIXED to describe the variances and covariances of mu attenuation. All models included a random intercept for each individual. Of the 48 participants, there were 36 unique families enrolled in this study. To account for possible shared variance between family members, statistical analyses included random intercepts for each family. Post hoc comparisons were conducted using least squares differences. Significant effects are presented for $p<.05$; marginal effects are presented for $p=.06$ to .1 .

Two different strategies were implemented. First, overall group mu attenuation differences were assessed as an average value across all epochs for each condition (model 0, model 1). Effects of condition were separated by context $(0=$ nonsocial, $1=$ social $)$ and environment ( 0 = live action, 1 = computer generated). Additional subject predictors were included in model 1 as fixed effects to test additional contribution by each individual's age and gender, particularly as carrier and typical groups consisted of both children and adults. Age was centered at 9 years due to the fact that the majority of individuals were between 6 and 18 years of age $(n=36)$ with an average age of 8.6 years. A categorical binary variable were used for gender $(0=$ male, $1=$ female $)$. In addition, a predictor describing the number of epochs contributed by each individual was added as a fixed effect to determine whether the results were influenced by the overall amount of data for each person. Effects were added simultaneously initially to model 0 , and nonsignificant effects were removed to generate model 1 .

Second, we were also interested in whether mu attenuation varied across the course of the video. To validate the necessity for this analysis, interclass correlations calculated from an empty model indicated that $46.7 \%$ of the variance for mu attenuation is due to overall withinperson effects, while the majority of variance $(53.3 \%)$ is due to trial order. This indicates that mu attenuation is related to changes across the course of the video, such that a fixed effect of time (e.g., temporal order for each epoch) merits addition to the multilevel models. Time was centered at epoch 30 (i.e., the 30th epoch surviving epoch rejection due to artifacts). Thus, model 2 included predictors from model 1 with time added as a fully interacting predictor with context, environment, and group.

\section{Results}

Averaged data (model 0)

A full factorial design between context, environment, and group with age, gender, and number of epoch predictors was estimated in model 0 . First, age significantly contributed to model $0, F(1,31.9)=5.11, p=0.031$, suggesting that older individuals are predicted to exhibited incrementally greater $\mathrm{mu}$ attenuation $(0.015$ more mu attenuation for every year older than 9 years). Second, mu attenuation was not affected by gender, $F(1,40.9)=0.23, p=0.62$, indicating that female and male individuals exhibit equivalent patterns of mu attenuation within groups and across conditions. Third, the number of epochs contributed by each individual also did not contribute to patterns of mu attenuation, $F(1,40.9)=0.42, p=0.52$. This was important to determine since the ASD group contributed fewer trials due to having more trials rejected by artifacts (30.8\%) than any of the other groups (deletion carriers, $14.4 \%$; duplication carriers, $11.3 \%$; typical, $9.7 \%$ ). Thus, gender and number of epoch predictors were subsequently removed for model 1.

\section{Averaged data (model 1)}

Topographic maps representing group differences in power are presented in Fig. 3 for each stimulus context (collapsed across stimulus environment). In the final model 1, all fixed effects were significant $(p<0.05$, uncorrected) with the exception of a main effect of the group. Model 1 results are reported in Table 2. Similar to model 0 , age significantly contributed to the model, indicating increased $\mathrm{mu}$ attenuation for older individuals (0.016 more mu attenuation for every year older than 9 years). Main effects of context and environment indicated more mu attenuation for nonsocial (compared to social) conditions and live action (compared to computer generated) conditions. These effects were modulated by a context $\times$ environment interaction, indicating that computer-generated conditions elicited more mu attenuation for nonsocial conditions, $t(10000)=4.43, p<0.0001$.

The primary aim of this paper was to address group differences across conditions. Figure 4 depicts observed mu attenuation by group across all conditions (context $\times$ environment). There was no main effect of a group, indicating that each of the four groups had overall similar levels of mu attenuation. However, we observed a significant interaction between group and context with three different patterns of relative mu attenuation between social and nonsocial motion. First, only the typical group elicited the predicted pattern with more $\mathrm{mu}$ attenuation to social than nonsocial conditions, $t(10,000)=2.30, p=0.022$. Second, both of the $16 p 11.2$ carrier groups exhibited the opposite pattern of mu attenuation, such that these groups elicited more $\mathrm{mu}$ attenuation to nonsocial than social conditions [deletion carriers, $t(10,000)=4.92, p<0.0001$; duplication carriers $t(10,000)=2.00, p=0.046]$. Third, the ASD group did not differentiate between conditions, $t(10,000)=1.43$, $p=0.15$. 


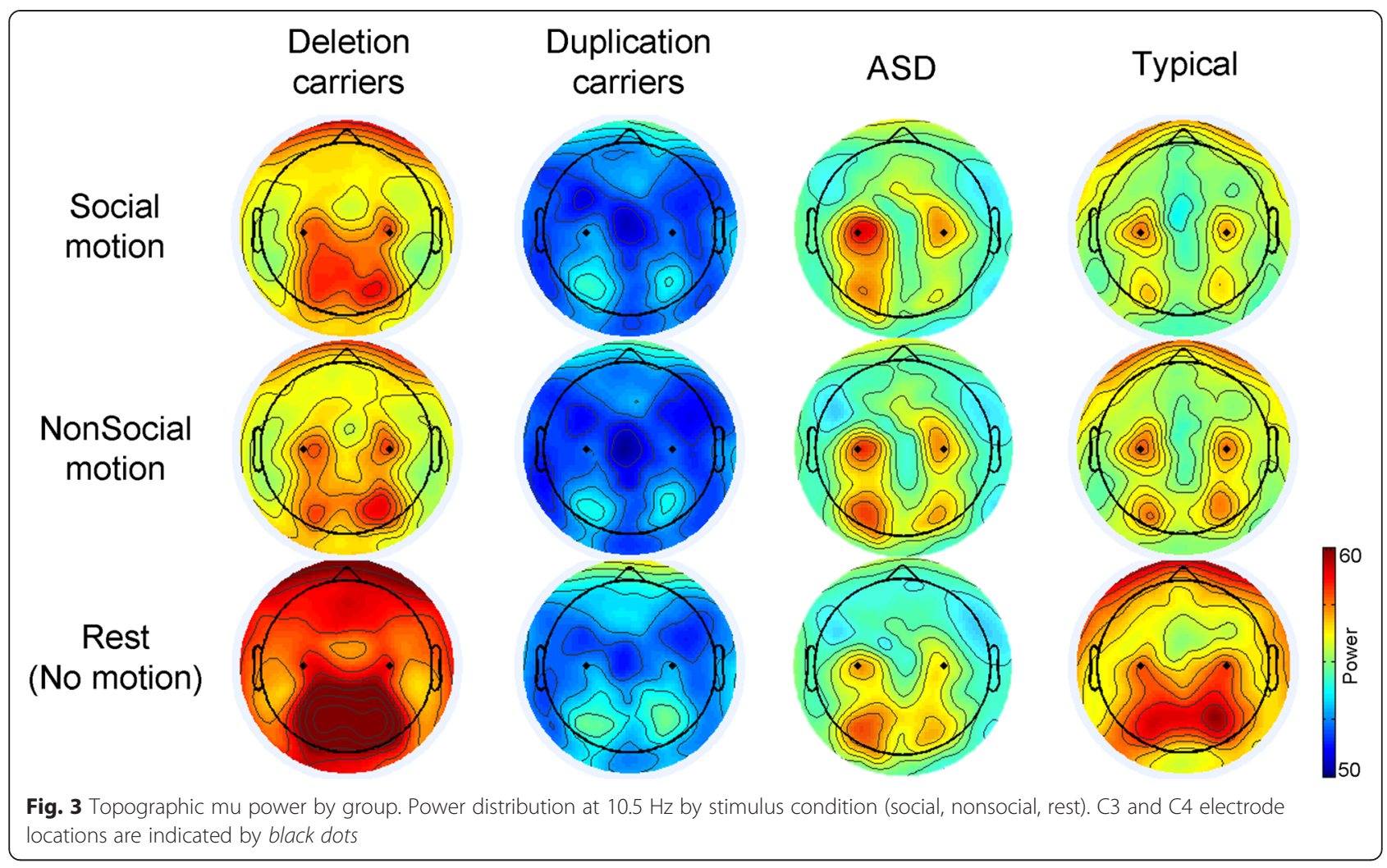

The group $\times$ environment interaction was qualified by the three-way interaction between group, context, and environment. This interaction indicated that the effect of more social mu attenuation than nonsocial for the typical group was primarily driven by differences within the live action environment, $t(10,000)=2.80, p=.0051$, but not the computer-generated environment, $t(10,000)=0.45$, $p=0.65$. The opposite was true for the deletion carriers, in which the nonsocial context differences (more nonsocial $\mathrm{mu}$ attenuation than social) were primarily driven by differences within the computer-generated environment, $t(10,000)=6.52, p<0.0001$, but not the live action environment, $t(10,000)=0.38, p=0.70$. Duplication carriers and the ASD group did not exhibit context discrimination differences between environments.

Table 2 Model 1 results

\begin{tabular}{lllll}
\hline Fixed effects & $F$ & $d f_{\text {between }}$ & $d f_{\text {within }}$ & $p$ value \\
\hline Context & 10.54 & 1 & 10,000 & 0.0012 \\
Environment & 10.75 & 1 & 10,000 & 0.0010 \\
Context $\times$ environment & 9.3 & 1 & 10,000 & 0.0023 \\
Group & 0.98 & 3 & 42 & 0.4129 \\
Group $\times$ context & 9.45 & 3 & 10,000 & $<.0001$ \\
Group $\times$ environment & 5.45 & 3 & 10,000 & 0.0010 \\
Group $\times$ context $\times$ environment & 3.46 & 3 & 10,000 & 0.0156 \\
Age & 7.97 & 1 & 31.9 & 0.0081 \\
\hline
\end{tabular}

\section{Single-trial analysis (model 2)}

Results for model 2 are reported in Table 3, and parameter estimates are reported in Table 4 . Negative parameter estimates reflect more mu attenuation. Parameter estimates are described in relation to the intercept (i.e., all fixed

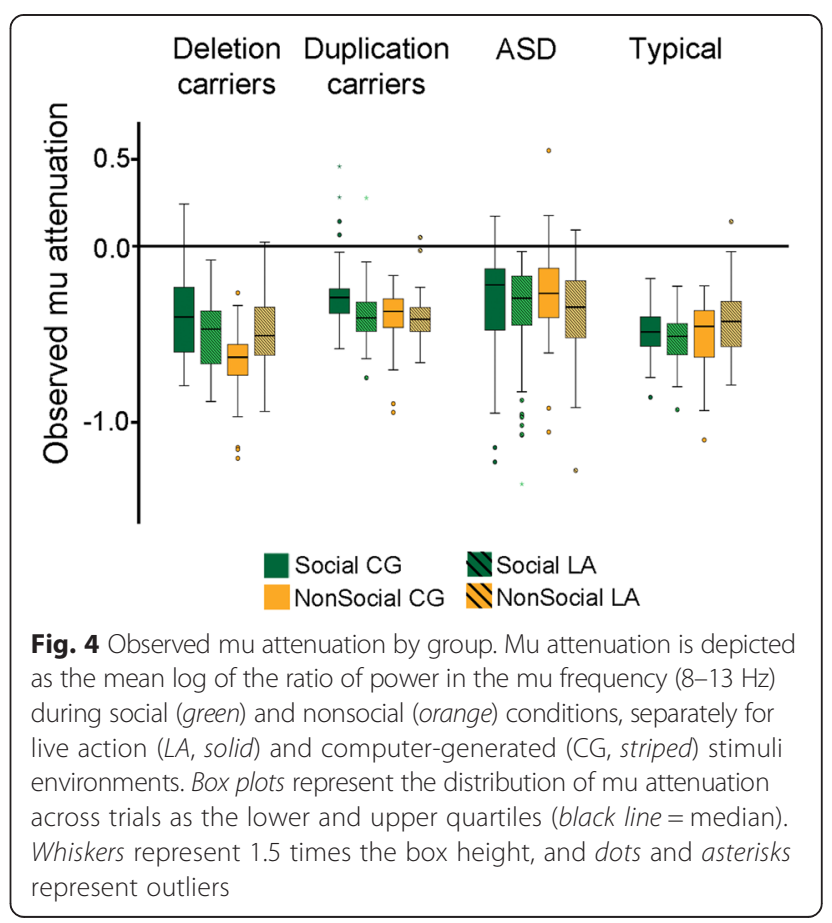


Table 3 Model 2 results

\begin{tabular}{lllll}
\hline Fixed effects & $F$ & $d f_{\text {between }}$ & $d f_{\text {within }}$ & $p$ value \\
\hline Context & 6.74 & 1 & 10,000 & 0.0094 \\
Environment & 9.38 & 1 & 10,000 & 0.0022 \\
Context $\times$ environment & 4.41 & 1 & 10,000 & 0.0357 \\
Group & 0.97 & 3 & 42.1 & 0.418 \\
Group $\times$ context & 8.41 & 3 & 10,000 & $<.0001$ \\
Group $\times$ environment & 4.78 & 3 & 10,000 & 0.0025 \\
Group $\times$ context $\times$ environment & 3.24 & 3 & 10,000 & 0.0213 \\
Age & 8.6 & 1 & 32.7 & 0.0061 \\
Time & 101.57 & 1 & 10,000 & $<.0001$ \\
Time $\times$ context & 0.02 & 1 & 10,000 & 0.8926 \\
Time $\times$ environment & 5.58 & 1 & 10,000 & 0.0182 \\
Time $\times$ context $\times$ environment & 0.47 & 1 & 10,000 & 0.4908 \\
Time $\times$ group & 9.97 & 3 & 10,000 & $<.0001$ \\
Time $\times$ group $\times$ context & 2.35 & 3 & 10,000 & 0.0705 \\
Time $\times$ group $\times$ environment & 1.84 & 3 & 10,000 & 0.1377 \\
Time $\times$ group $\times$ context $\times$ & 1.48 & 3 & 10,000 & 0.2181 \\
environment & & & & \\
\hline
\end{tabular}

effects at 0), which represents the expected mu attenuation for a 9-year-old in the typical group during the 30 th epoch in the live action nonsocial condition. For example, model 2 estimated 0.0569 more mu attenuation in the social context and 0.0072 less mu attenuation in the computer-generated environment for a 9-year-old typical subject. Similar to model 0 and model 1, age was associated with increased $\mathrm{mu}$ attenuation for individuals older than 9 years of age, consistent with prior research [1, 2, 24-30, 37].

Main effects of condition and group estimated at epoch 30 were similar to the overall effects of model 1 with one exception. Unlike model 1 , which predicted that more mu attenuation for the social live action condition, model 2 predicted no difference between live action and computergenerated environments, $t(10,000)=0.36, \quad p=0.72$. This suggests that model 1 indicated that differences in $\mathrm{mu}$ attenuation for certain environments in the typical and deletion carrier groups may resolve over the course of the experiment by epoch 30 , such that there is no longer a difference in mu attenuation by environment.

A main effect of time indicated that for subsequent epochs, the mu attenuation intercept (i.e., typical 9-yearold watching a live action nonsocial video) was expected to decrease or lessen. However, as described by the significant group $\times$ time interaction, the rate of mu attenuation lessening differed by group. The first column in Fig. 5 depicts this effect as the grand average of observed $\mathrm{mu}$ attenuation over epochs for each of the groups. Compared to the typical slope (0.0048), mu attenuation decreased over time more rapidly for the deletion carriers (slope $=0.0028)$ and less rapidly for the duplication carriers (slope $=.0065$ ). In contrast to the other three groups, the ASD slope increased in mu attenuation (slope $=-0.00022$ ), suggesting that the neural network supporting motion perception may be increasing efficiency over the course of the videos.

Although the time $\times$ group $\times$ context interaction was marginally significant ( $p=.071$, see Table 3$)$, the second and third columns in Fig. 5 depict the change in mu attenuation for social and nonsocial conditions for computer-generated and live action environments, respectively. These plots highlight that group differences in context discrimination are more apparent during increased exposure to the stimuli (e.g., after epoch 30 of video viewing). Importantly, this figure also illustrates that context discrimination can largely be described as the relative rate of $\mathrm{mu}$ attenuation lessening for social and nonsocial motion. For instance, model 1 indicated larger context discrimination for the deletion carriers in the computer-generated motion. Looking at the patterns of mu attenuation over time, this effect is largely driven by the rapid decreasing mu attenuation of the social condition, whereas mu attenuation for the nonsocial condition does not lessen much over time.

To fulfill our objective of characterizing dynamic changes in mu attenuation related to social perception, we conducted a series of pairwise comparisons between social and nonsocial context for each group every 5 epochs from epoch 1 to epoch 60. Figure 6 plots mu attenuation modulation as a difference score, such that negative values represent more mu attenuation to social than nonsocial context. Each group exhibited a unique pattern of mu attenuation related to context discrimination. Both deletion and duplication carriers exhibited more nonsocial than social $\mathrm{mu}$ attenuation. This pattern was consistent for deletion carriers from trial $1[t \mathrm{~s}(10,000)=2.01-4.22$, $p$ s $<0.05$ ] until epoch 60, $t(10,000)=1.73, p=0.083$. However, the duplication carriers did not begin discriminating contexts until epoch 30 and continued to exhibit more $\mathrm{mu}$ attenuation for nonsocial conditions until the end of the video, $t \mathrm{~s}(10,000)=2.09-2.92$, $p \mathrm{~s}<0.05$. In contrast to the carrier groups, the typical group exhibited greater $\mathrm{mu}$ attenuation for social conditions at epoch 25 , ts $(10,000)=2.0-2.6, p<0.05$. Unlike all other groups, the ASD group did not exhibit mu attenuation context discrimination at any epoch, indicating equivalent levels of $\mathrm{mu}$ attenuation for social and nonsocial conditions.

\section{Post hoc comparisons of $16 p 11.2$ carriers}

To better understand how these patterns may be related to heterogeneity within these unique samples, we conducted a series of post hoc ANOVAs to determine if patterns of mu attenuation are consistent for $16 p 11.2$ carriers 
Table 4 Model 2 estimates

\begin{tabular}{|c|c|c|c|c|}
\hline Parameter Estimates & Estimated additional categorical effect & Comparison categorical effect & Estimate & Standard error \\
\hline Intercept & & & -0.1886 & 0.2293 \\
\hline Context & Biological & Nonbiological & -0.0569 & 0.0218 \\
\hline Environment & Computer generated & Live action & 0.0072 & 0.0222 \\
\hline Context $\times$ environment & Biological, computer generated & Biological, live action & 0.0351 & 0.0310 \\
\hline \multirow[t]{3}{*}{ Group } & Deletion & Typical & 0.0087 & 0.1603 \\
\hline & Duplication & Typical & 0.1623 & 0.1655 \\
\hline & ASD & Typical & 0.1223 & 0.1806 \\
\hline \multirow[t]{3}{*}{ Group $\times$ context } & Biological, deletion & Biological, typical & 0.0637 & 0.0335 \\
\hline & Biological, duplication & Biological, typical & 0.0935 & 0.0335 \\
\hline & Biological, ASD & Biological, typical & 0.0897 & 0.0400 \\
\hline \multirow[t]{3}{*}{ Group $\times$ environment } & Computer generated, deletion & Live action, typical & -0.1006 & 0.0348 \\
\hline & Computer generated, duplication & Live action, typical & 0.0634 & 0.0345 \\
\hline & Computer generated, ASD & Live action, typical & 0.0454 & 0.0425 \\
\hline \multirow[t]{3}{*}{ Group $\times$ context $\times$ environment } & Biological, computer generated, deletion & Biological, live action, typical & 0.1045 & 0.0480 \\
\hline & Biological, computer generated, duplication & Biological, live action, typical & -0.0328 & 0.0476 \\
\hline & Biological, computer generated, ASD & Biological, live action, typical & -0.0501 & 0.0576 \\
\hline Age & & & -0.0164 & 0.0056 \\
\hline Time & & & 0.0048 & 0.0009 \\
\hline Time $\times$ context & Biological & Nonbiological & -0.0001 & 0.0013 \\
\hline Time $\times$ environment & Computer generated & Live action & -0.0015 & 0.0014 \\
\hline Time $\times$ context $\times$ environment & Biological, computer generated & Biological, Live action & -0.0014 & 0.0018 \\
\hline \multirow[t]{3}{*}{ Time $\times$ group } & Deletion & Typical & 0.0017 & 0.0014 \\
\hline & Duplication & Typical & -0.0020 & 0.0014 \\
\hline & ASD & Typical & -0.0051 & 0.0017 \\
\hline \multirow[t]{3}{*}{ Time $\times$ group $\times$ context } & Biological, deletion & Biological, typical & -0.0019 & 0.0020 \\
\hline & Biological, duplication & Biological, typical & 0.0034 & 0.0020 \\
\hline & Biological, ASD & Biological, typical & -0.0001 & 0.0025 \\
\hline \multirow[t]{3}{*}{ Time $\times$ group $\times$ environment } & Computer generated, deletion & Live action, typical & -0.0010 & 0.0021 \\
\hline & Computer generated, duplication & Live action, typical & -0.0007 & 0.0021 \\
\hline & Computer generated, ASD & Live action, typical & 0.0039 & 0.0026 \\
\hline \multirow[t]{3}{*}{ Time $\times$ group $\times$ context $\times$ environment } & Biological, computer generated, deletion & Biological, live action, typical & 0.0046 & 0.0028 \\
\hline & Biological, computer generated, duplication & Biological, live action, typical & -0.0006 & 0.0028 \\
\hline & Biological, computer generated, ASD & Biological, live action, typical & -0.0018 & 0.0035 \\
\hline
\end{tabular}

between (1) children and adults and (2) between 16p11.2 carriers with and without an ASD diagnosis. While these comparisons may be subjected to type I and II errors due to small sample size, these analyses were meant to qualitatively support model 1 and model 2 predictions. The patterns of $\mathrm{mu}$ attenuation for these comparisons are depicted in Additional file 1: Figure S1, Additional file 2: Figure S2, and Additional file 3: Figure S3.

First, child deletion carriers $[n=9, F(1,1861)=16.28$, $p<.001]$ and adult duplication carriers $[n=5, F(1,1111)=$ $15.19, p<.001]$ exhibited the overall group pattern exhibited with greater $\mathrm{mu}$ attenuation for nonsocial (compared to social) conditions. There was no difference between context for adult deletion carriers $[n=7, F(1$, $740)=1.87, p=.17]$ or child duplication carriers $[n=3$, $F(1,1567)=.002, p=.96]$. However, visual inspection of the dynamic mu attenuation patterns across time (Additional file 2: Figure S2) suggests that the adult deletion carriers exhibited greater mu attenuation for the nonsocial context. Unlike the other $16 p 11.2$ carrier age groups, child duplication carriers exhibited a pattern of mu attenuation changes that is more similar to the ASD group (no context discrimination but trending towards social greater than nonsocial). 


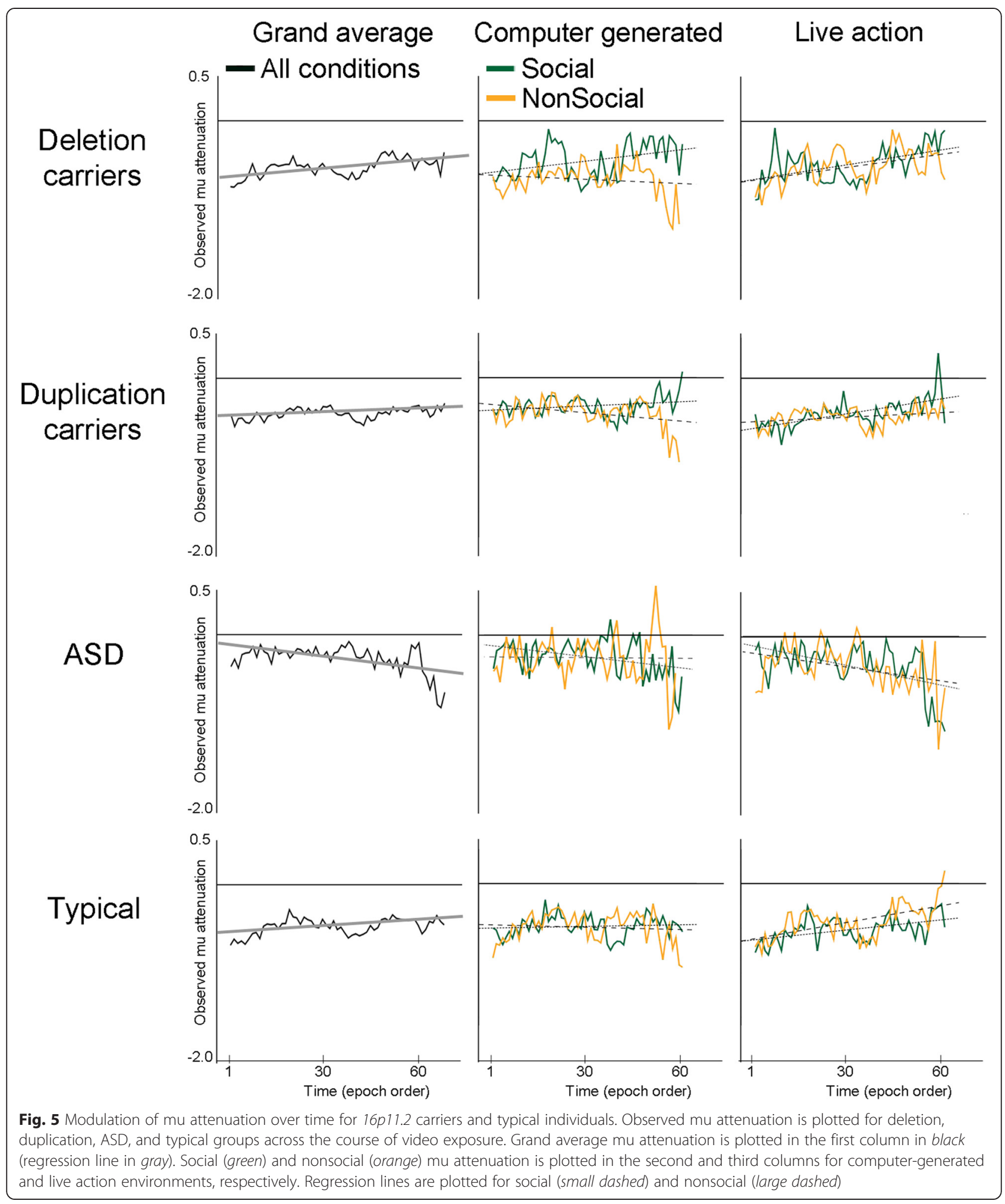

Second, neither deletion carriers $(n=3)$ nor duplication carriers $(n=3)$ with an ASD diagnosis exhibited context discrimination, $F \mathrm{~s}<.31, p \mathrm{~s}>.58$, similar to the pattern of mu attenuation for the ASD (idiopathic, non- 16p11.2 carrier) group. Visual inspection of the dynamic $\mathrm{mu}$ attenuation patterns across time (Additional file 3: Figure S3) indicates a large amount of variability for the deletion carriers with ASD, consistent with prior work looking 


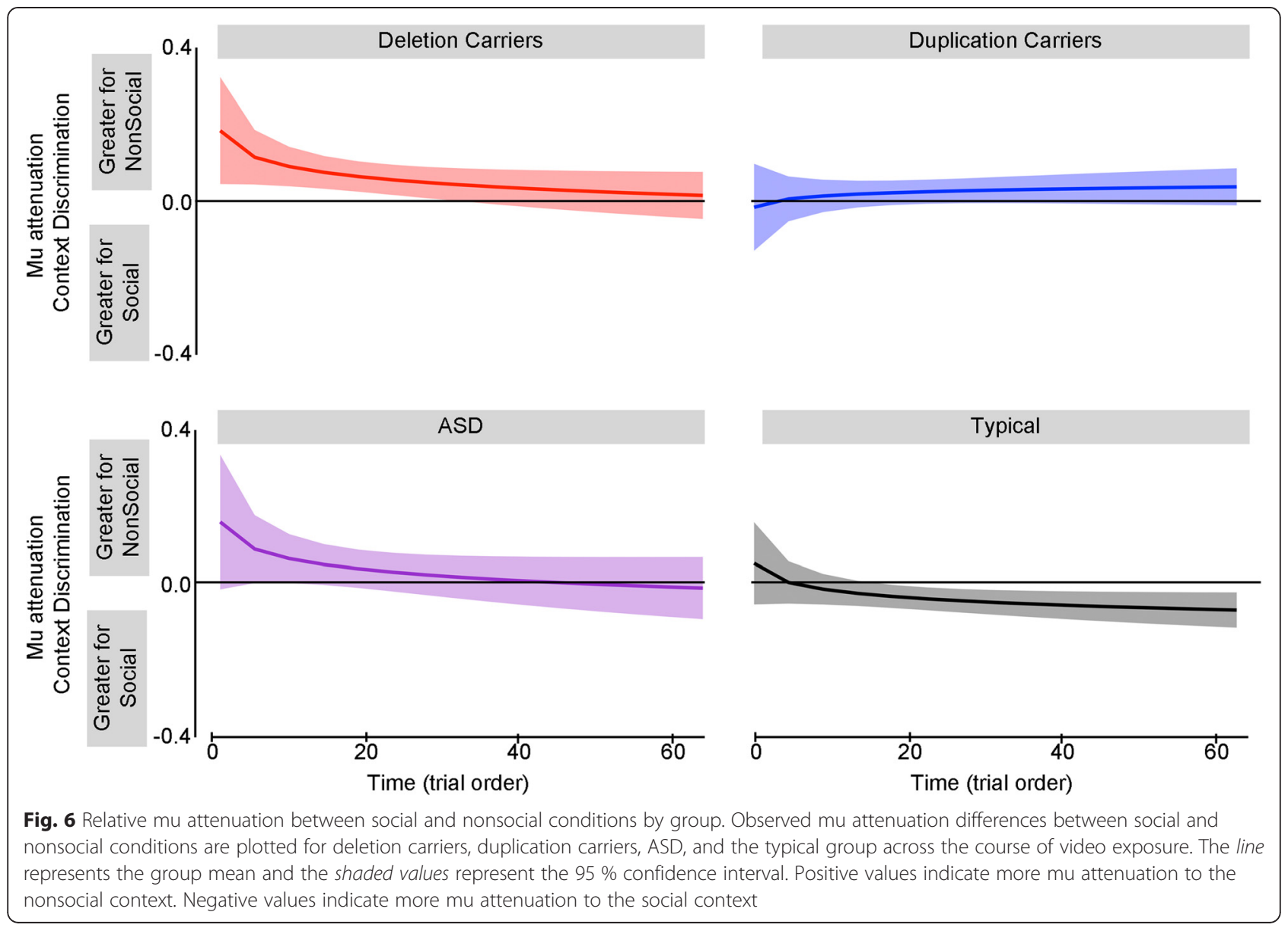

at social motion perception in ASD [3, 5-14, 22, 31]. These results suggest that social motion mu attenuation within 16p11.2 carriers is associated with ASD and the concomitant social cognitive phenotype.

\section{Discussion}

This study sought to characterize how the functional social brain phenotype of a preliminary sample of individuals with 16p11.2 $\mathrm{CNVs}$ compares to typical and ASD individuals. As predicted, typical individuals exhibited greater mu attenuation for social motion relative to nonsocial motion, particularly within the live action environment. Contrary to this typical pattern of mu attenuation, we found that both 16p11.2 CNV duplication and deletion carriers exhibited greater mu attenuation for nonsocial motion relative to social motion. This pattern differed from individuals with ASD, who elicited equivalent levels of mu attenuation across conditions, consistent with prior work indicating reduced $\mathrm{mu}$ attenuation in ASD in response to social motion compared to controls [15, 32-35, 37, 38].

An innovation and benefit of our analytic strategy was the use of single-trial analysis to assess ongoing dynamic changes over the course of the motion observation, designating two important discoveries. First, the results confirm that mu attenuation is not static but rather decreases or lessens across exposure to motion stimuli for the typical and 16p11.2 CNV groups. Specifically, this decrease in mu attenuation occurs more rapidly for the deletion carriers than either the duplication carriers or the typical group, whereas mu attenuation increases over time for the ASD group. The degree to which the mu rhythm attenuates overexposure to stimuli may implicate different patterns of general habituation or information processing. Recent evidence suggests that $16 p 11.2$ CNVs exhibit a dose-dependent effect on both cortical and subcortical structures [46], such that compared to controls, brain volume is increased for deletion carriers and reduced for duplication carriers. Thus, the discordant rates of habituation observed among deletion and duplication carriers may correspond to differences in brain structure and/or circuitry.

Second, the single-trial analysis indicated that the relative patterns of mu attenuation between social and nonsocial motion vary differently for each group over time. Specifically, these patterns indicated three unique atypical 
patterns of mu attenuation: overall increase for nonsocial (16p11.2 deletion), mid-experiment increase for nonsocial (16p11.2 duplication), and no motion discrimination (ASD). The duplication carriers and the typical group exhibited proportionally equivalent $\mathrm{mu}$ attenuation during the initial portion of social and nonsocial videos. Subsequently, after 25-30 epochs, the modulation of mu attenuation diverged for these two groups, such that the duplication carriers followed an atypical trajectory with greater mu attenuation for nonsocial conditions. It may be that while social stimuli are initially similarly salient for duplication carriers and typical individuals, the maintenance of that salience at the neurophysiological level differs, resulting in a rapid decrease in neurophysiological processes devoted to social information processing.

In contrast, discrimination is stable across exposure for the $16 p 211.2$ deletion carriers, albeit with an atypical pattern of $\mathrm{mu}$ attenuation (i.e., greater attenuation for nonsocial). While both CNV groups exhibit greater mu attenuation for nonsocial relative to social motion, this overall pattern of discrimination occurs during the later exposure trials for the duplication carriers. This finding reflects different temporal processing trajectories, which may be driven by unique underlying neural systems for integrating social information. One possible explanation may be that the CNV groups differ in level of motivation to engage with social stimuli. Considering the rapid rate of $\mathrm{mu}$ attenuation reduction during the observation of social motion for the deletion group (see Fig. 4), it is possible that deletion carriers may fail to truly engage with the social stimuli, driven perhaps by less motivation or salience.

However, within this preliminary study, individuals with 16p11.2 CNVs and a diagnosis of ASD have divergent trajectories (compared to 16p11.2 carriers without ASD), more aligned with the individuals with idiopathic ASD. In other words, the functional social brain phenotype associated with ASD supersedes the unique 16p11.2 deletion and 16p11.2 duplication phenotype. Each unique atypical pattern may correspond to different aspects of social cognitive impairments. For instance, individuals with ASD do not exhibit social and nonsocial motion discrimination, supporting theories of complex information dysfunction $[42,52]$. In comparison, the CNV carriers without ASD respond more strongly to nonsocial motion, possibly indicative of a reduction in salience or motivation for social information or increased salience of nonsocial information. Considering the social motion perception pattern for individuals with $16 p 11.2 \mathrm{CNVs}$ and ASD, it is likely that concomitant features of social cognition (e.g., emotion recognition, social motivation) more closely resemble ASD impairments [53-56].

Given the relationship between 16p11.2 CNV and neurodevelopmental disorders, a developmental model is critical.
Prior work indicates a diagnosis-independent emergence of mu attenuation across development [37], but both aspects of time (developmental stage and temporal processing) are critical in understanding how the functional social brain phenotype emerges. While both duplication and deletions carriers showed specific effects of neural responses to social motion, it is possible that the observed variability within our carrier groups may have been related to "second hits" in the genome or to genetic variation at other loci $[57,58]$. In the same vein, the "idiopathic" ASD group likely reflects a combination of etiologies, which may separately confer varying effects on the neurophysiological presentation rendering increased variability within that group.

The three primary strengths of this study include the use of careful molecular subtyping as a "genetics-first" approach [59], neurodevelopmental comparisons between CNV and ASD groups, and the unique statistical methods that permitted the dynamic measurement of social perception. Nevertheless, this study is limited by sample size and age range of the individuals. Our post hoc comparisons suggest that social perception $16 p 11.2$ CNV patterns are fairly consistent across development, despite the large age range. Considering the rarity of this group of individuals, the results are informative as a preliminary investigation of the $16 p 11.2 \mathrm{CNV}$ social brain phenotype. As genetic testing becomes more commonplace, the percentage of known individuals with $16 p 11.2$ $\mathrm{CNVs}$ will increase. While investigating this $\mathrm{CNV}$ and other rare variants is a long-term process, these results justify continuing to use neuroimaging in order to elucidate the underlying pathogenic mechanisms involved with unique molecular subtypes.

\section{Conclusions}

In conclusion, our findings strongly support the notion that $16 p 11.2$ carriers exhibit atypical social perception, similar to, yet distinct from, ASD. Future research should continue to address clinical and behavioral heterogeneity by reducing variance through large, carefully matched populations. The process by which these patterns emerge in relation to developmental age may be of particular interest, as well as how mu attenuation is impacted by clinical interventions promoting social development. Additional considerations for individual differences would benefit from the inclusion of robust behavioral measures of social cognition previously linked to spectral power in the mu rhythm, such as imitative ability [60].

\section{Additional files}

Additional file 1: Figure S1. Post hoc comparisons of age and ASD Diagnosis within 16p11.2 carriers. Error bars represent $95 \%$ confidence interval of the mean. Significant comparisons are noted with an asterisk. 
Additional file 2: Figure S2. Relative mu attenuation between social and nonsocial conditions over time for $16 p 11.2$ carriers by age group. Observed mu attenuation differences between social and nonsocial conditions are plotted for deletion carriers and duplication carriers, separately for children (younger than 18 years, dash-dot line) and adults (18 years and older, solid line). The line represents the group mean and the shaded values represent the $95 \%$ confidence interval. Positive values indicate more mu attenuation to the nonsocial context. Negative values indicate more mu attenuation to the social context. Black arrows indicate the point in time by which model 2 indicated significant context differences. Gray arrows with an " $X$ " indicate the point in which there are no longer a significant context difference.

Additional file 3: Figure S3. Relative mu attenuation between social and nonsocial conditions over time for $16 p 11.2$ carriers by ASD diagnosis. Observed mu attenuation differences between social and nonsocial conditions are plotted for deletion carriers and duplication carriers, separately for individuals without an ASD diagnosis (top, solid line) and with an ASD diagnosis (bottom, dashed line). The line represents the group mean and the shaded values represent the $95 \%$ confidence interval. Positive values indicate more mu attenuation to the nonsocial context. Negative values indicate more mu attenuation to the social context. Black arrows indicate the point in time by which model 2 indicated significant context differences. Gray arrows with an " $X$ " indicate the point in which there are no longer a significant context difference.

\section{Competing interests}

The authors declare that they have no competing interests.

\section{Authors' contributions}

$\mathrm{CH}$ and TD conducted data processing and analysis. AK, BA, SJW, and RAB conceived of the study, participated in design and coordination of research participants, and conducted data processing and analysis. All authors discussed results interpretations, completed manuscript preparation, and read and approved the final manuscript.

\section{Acknowledgements}

We are grateful for the families and individuals who volunteered to participate in this study. Research reported in this publication was supported by the Eunice Kennedy Shriver National Institute Of Child Health and Human Development of the National Institutes of Health (U54HD083091 Bernier), National Institutes of Mental Health (MH100047 Bernier; MH10028 Webb/Bernier), and the Simons Foundation.

\section{Author details}

'Department of Psychiatry and Behavioral Sciences, University of Washington, 1959 Northeast Pacific Street \#115, Seattle, WA 98195, USA. 'Seattle Children's Research Institute, 2001 8th Avenue \#400, Seattle, WA 98121, USA.

\section{Received: 26 January 2015 Accepted: 19 June 2015} Published online: 24 July 2015

\section{References}

1. Weiss LA, Shen Y, Korn JM, Arking DE, Miller DT, Fossdal R, et al. Association between microdeletion and microduplication at 16p11.2 and autism. N Engl J Med. 2008;358:667-75.

2. Marshall CR, Noor A, Vincent JB, Lionel AC, Feuk L, Skaug J, et al. Structural variation of chromosomes in autism spectrum disorder. Am J Hum Genet. 2008;82:477-88.

3. Kumar RA, KaraMohamed S, Sudi J, Conrad DF, Brune C, Badner JA, et al. Recurrent 16p11.2 microdeletions in autism. Hum Mol Genet. 2008;17:628-38

4. Horev G, Ellegood J, Lerch JP, Son Y-EE, Muthuswamy L, Vogel H, et al. Dosage-dependent phenotypes in models of 16p11.2 lesions found in autism. Proc Natl Acad Sci U S A. 2011;108:17076-81.

5. Jacquemont S, Reymond A, Zufferey F, Harewood L, Walters RG, Kutalik Z, et al. Mirror extreme BMI phenotypes associated with gene dosage at the chromosome 16p11.2 locus. Nature. 2011;478:97-102.

6. Bardakjian TM, Kwok S, Slavotinek AM, Schneider AS. Clinical report of microphthalmia and optic nerve coloboma associated with a de novo microdeletion of chromosome 16p11.2. Am J Med Genet A. 2010;152A:3120-3.

7. Bijlsma EK, Gijsbers ACJ, Schuurs-Hoeijmakers JHM, van Haeringen A, van de Putte DEF, Anderlid BM, et al. Extending the phenotype of recurrent rearrangements of 16p11.2: deletions in mentally retarded patients without autism and in normal individuals. Eur J Med Genet. 2009;52:77-87.

8. Dale RC, Grattan-Smith P, Fung VSC, Peters GB. Infantile convulsions and paroxysmal kinesigenic dyskinesia with 16p11.2 microdeletion. Neurology. 2011;77:1401-2.

9. Fernandez BA, Roberts W, Chung B, Weksberg R, Meyn S, Szatmari P, et al. Phenotypic spectrum associated with de novo and inherited deletions and duplications at 16p11.2 in individuals ascertained for diagnosis of autism spectrum disorder. J Med Genet. 2010;47:195-203.

10. Hanson E, Nasir RH, Fong A, Lian A, Hundley R, Shen $Y$, et al. Cognitive and behavioral characterization of 16p11.2 deletion syndrome. J Dev Behav Pediatr. 2010;31:649-57.

11. Puvabanditsin S, Nagar MS, Joshi M, Lambert G, Garrow E, Brandsma E. Microdeletion of 16p11.2 associated with endocardial fibroelastosis. Am J Med Genet A. 2010;152A:2383-6.

12. Rosenfeld JA, Coe BP, Eichler EE, Cuckle H, Shaffer LG. Estimates of penetrance for recurrent pathogenic copy-number variations. Genet Med. 2012;15:478-81.

13. Shimojima K, Inoue T, Fujii Y, Ohno K, Yamamoto T. A familial 593-kb microdeletion of $16 \mathrm{p} 11.2$ associated with mental retardation and hemivertebrae. Eur J Med Genet. 2009;52:433-5.

14. Walsh KM, Bracken MB. Copy number variation in the dosage-sensitive 16 p11.2 interval accounts for only a small proportion of autism incidence: a systematic review and meta-analysis. Genet Med. 2011;13:377-84.

15. Zufferey F, Sherr EH, Beckmann ND, Hanson E, Maillard AM, Hippolyte L, et al. A $600 \mathrm{~kb}$ deletion syndrome at 16p11.2 leads to energy imbalance and neuropsychiatric disorders. J Med Genet. 2012;49:660-8.

16. Moreno-De-Luca D, Moreno-De-Luca A, Cubells JF, Sanders SJ. Cross-disorder comparison of four neuropsychiatric CNV loci. Current Genetic Medicine Reports. 2014;2:151-61.

17. Hanson E, Bernier R, Porche K, Jackson Fl, Goin-Kochel RP, Green Snyder LA, et al. The cognitive and behavioral phenotype of the 16p11.2 deletion in a clinically ascertained population. Biol Psychiatry. 2014;52:77-87.

18. Wing $L$, Gould J. Severe impairments of social interaction and associated abnormalities in children: epidemiology and classification. J Autism Dev Disord. 1979;9:11-29.

19. Kaiser MD, Hudac CM, Shultz S, Lee SM, Cheung C, Berken AM, et al. Neural signatures of autism. Proc Natl Acad Sci. 2010;107:21223-8.

20. Meltzoff AN, Decety J. What imitation tells us about social cognition: a rapprochement between developmental psychology and cognitive neuroscience. Philos Trans R Soc Lond B Biol Sci. 2003;358:491-500.

21. Allison T, Puce A, McCarthy G. Social perception from visual cues: role of the STS region. Trends Cogn Sci. 2000;4:267-78.

22. Pelphrey KA, Shultz S, Hudac CM, Vander Wyk BC. Research review: constraining heterogeneity: the social brain and its development in autism spectrum disorder. J Child Psychol Psychiatry. 2011;52:631-44.

23. Freitag CM, Konrad C, Häberlen M, Kleser C, von Gontard A, Reith W, et al. Perception of biological motion in autism spectrum disorders. Neuropsychologia. 2008:46:1480-94.

24. Pfurtscheller G, Neuper C, Andrew C, Edlinger G. Foot and hand area mu rhythms. Int J Psychophysiol. 1997;26:121-35.

25. Arroyo S, Lesser RP, Gordon B, Uematsu S, Jackson D, Webber R. Functional significance of the mu rhythm of human cortex: an electrophysiologic study with subdural electrodes. Electroencephalogr Clin Neurophysiol. 1993;87:76-87.

26. Babiloni C, Babiloni F, Carducci F, Cincotti F, Cocozza G, Del Percio C, et al. Human cortical electroencephalography (EEG) rhythms during the observation of simple aimless movements: a high-resolution EEG study. Neuroimage. 2002;17:559-72.

27. Babiloni C, Carducci F, Cincotti F, Rossini PM, Neuper C, Pfurtscheller G, et al. Human movement-related potentials vs desynchronization of EEG alpha rhythm: a high-resolution EEG study. Neuroimage. 1999;10:658-65.

28. Muthukumaraswamy SD, Johnson BW, McNair NA. Mu rhythm modulation during observation of an object-directed grasp. Cogn Brain Res. 2004;19:195-201.

29. Muthukumaraswamy SD, Johnson BW. Primary motor cortex activation during action observation revealed by wavelet analysis of the EEG. Clin Neurophysiol. 2004;115:1760-6. 
30. Cochin S, Barthelemy C, Roux S, Martineau J. Observation and execution of movement: similarities demonstrated by quantified electroencephalography. Eur J Neurosci. 1999;11:1839-42.

31. Pineda JA. The functional significance of mu rhythms: translating "seeing" and "hearing" into "doing". Brain Res Brain Res Rev. 2005;50:57-68.

32. lacoboni M. Imitation, empathy, and mirror neurons. Annu Rev Psychol. 2009;60:653-70.

33. Ulloa ER, Pineda JA. Recognition of point-light biological motion: mu rhythms and mirror neuron activity. Behav Brain Res. 2007;183:188-94.

34. Pineda JA, Hecht E. Mirroring and mu rhythm involvement in social cognition: are there dissociable subcomponents of theory of mind? Biol Psychiatry. 2009;1-9.

35. Bernier R, Dawson G, Webb S, Murias M. EEG mu rhythm and imitation impairments in individuals with autism spectrum disorder. Brain Cogn. 2007;64:228-37.

36. Dumas G, Soussignan R, Hugueville L, Martinerie J, Nadel J. Revisiting mu suppression in autism spectrum disorder. Brain Res. 2014;1585:108-19.

37. Oberman LM, McCleery JP, Hubbard EM, Bernier R, Wiersema JR, Raymaekers $R$, et al. Developmental changes in mu suppression to observed and executed actions in autism spectrum disorders. Soc Cogn Affect Neurosci. 2013:8:300-4

38. Oberman LM, Hubbard EM, McCleery JP, Altschuler EL, Ramachandran VS, Pineda JA. EEG evidence for mirror neuron dysfunction in autism spectrum disorders. Cogn Brain Res. 2005;24:190-8.

39. Tangwiriyasakul C, Verhagen R, van Putten MJAM, Rutten WLC. Importance of baseline in event-related desynchronization during a combination task of motor imagery and motor observation. J Neural Eng. 2013;10:026009.

40. Speirs SJ, Rinehart NJ, Robinson SR, Tonge BJ, Yelland GW. Efficacy of cognitive processes in young people with high-functioning autism spectrum disorder using a novel visual information-processing task. J Autism Dev Disord. 2014;44:2809-19.

41. Minshew NJ, Goldstein G. Autism as a disorder of complex information processing. Ment Retard Dev Disabil Res Rev 1998.

42. Williams DL, Goldstein G, Minshew NJ. Neuropsychologic functioning in children with autism: further evidence for disordered complex informationprocessing. Child Neuropsychol. 2006;12:279-98.

43. Kleinhans NM, Richards T, Weaver K, Johnson LC, Greenson J, Dawson G, et al. Association between amygdala response to emotional faces and social anxiety in autism spectrum disorders. Neuropsychologia. 2010;48:3665-70.

44. Swartz JR, Wiggins JL, Carrasco M, Lord C, Monk CS. Amygdala habituation and prefrontal functional connectivity in youth with autism spectrum disorders. J Am Acad Child Adolesc Psychiatry. 2013;52:84-93.

45. The Simons VIP Consortium. Simons Variation in Individuals Project (Simons VIP): a genetics-first approach to studying autism spectrum and related neurodevelopmental disorders. Neuron. 2012;73:1063-7.

46. Qureshi AY, Mueller S, Snyder AZ, Mukherjee P, Berman Jl, Roberts TPL, et al. Opposing brain differences in 16p11.2 deletion and duplication carriers. J Neurosci. 2014;34:11199-211.

47. Lord C, Rutter M, Le Couteur A. Autism Diagnostic Interview-Revised: a revised version of a diagnostic interview for caregivers of individuals with possible pervasive developmental disorders. J Autism Dev Disord. 1994;24:659-85.

48. Lord C, Rutter M, Goode S, Heemsbergen J, Jordan H, Mawhood L, et al. Austism diagnostic observation schedule: a standardized observation of communicative and social behavior. J Autism Dev Disord. 1989;19:185-212.

49. Wechsler D. Wechsler Abbreviated Scale of Intelligence (WASI). San Antonio, TX: The Psychological Corporation; 1999.

50. Elliott CD. Differential ability scales-LI. Pearson: San Antonio, TX; 2007.

51. Delorme A, Makeig S. EEGLAB: an open source toolbox for analysis of single-trial EEG dynamics including independent component analysis. J Neurosci Methods. 2004;134:9-21.

52. Happé FGE, Booth RDL. The power of the positive: revisiting weak coherence in autism spectrum disorders. Q J Exp Psychol. 2008;61:50-63.

53. Dawson G, Webb SJ, Carver L, Panagiotides H, McPartland J. Young children with autism show atypical brain responses to fearful versus neutral facial expressions of emotion. Dev Sci. 2004;7:340-59.

54. Lerner MD, McPartland JC, Morris JP. Multimodal emotion processing in autism spectrum disorders: an event-related potential study. Accid Anal Prev. 2012;1-11.

55. Kylliäinen A, Wallace S, Coutanche MN, Leppänen JM, Cusack J, Bailey AJ, et al. Affective-motivational brain responses to direct gaze in children with autism spectrum disorder. J Child Psychol Psychiatry. 2012;53:790-7.
56. Bellebaum C, Brodmann K, Thoma P. Active and observational reward learning in adults with autism spectrum disorder: relationship with empathy in an atypical sample. Cogn Neuropsychiatry. 2014;19:205-25.

57. Girirajan S, Brkanac Z, Coe BP, Baker C, Vives L, Vu TH, et al. Relative burden of large CNVs on a range of neurodevelopmental phenotypes. PLoS Genet. 2011;7, e1002334.

58. Girirajan S, Dennis MY, Baker C, Malig M, Coe BP, Campbell CD, et al. Refinement and discovery of new hotspots of copy-number variation associated with autism spectrum disorder. Am J Hum Genet. 2013;92:221-37.

59. Stessman HA, Bernier R, Eichler EE. A genotype-first approach to defining the subtypes of a complex disease. Cell. 2014;156:872-7.

60. Bernier R, Aaronson B, McPartland J. The role of imitation in the observed heterogeneity in EEG mu rhythm in autism and typical development. Brain Cogn. 2013;82:69-75

\section{Submit your next manuscript to BioMed Central and take full advantage of:}

- Convenient online submission

- Thorough peer review

- No space constraints or color figure charges

- Immediate publication on acceptance

- Inclusion in PubMed, CAS, Scopus and Google Scholar

- Research which is freely available for redistribution 